\title{
Fixed Coefficients for Certain Subclass of Univalent Functions using Hypergeometric Function
}

\author{
C. Selvaraj ${ }^{1}$, T. R. K. Kumar ${ }^{2}$, Thirupathi ${ }^{3}$ \\ ${ }^{1}$ Department of Mathematics, Presidency College, Chennai, Tamilnadu, India \\ ${ }^{2}$ Department of Mathematics, R.M.K. Engineering College, Tamilnadu, India \\ ${ }^{3}$ Department of Mathematics, R.M.K. Engineering College, Tamilnadu, India
}

\begin{abstract}
When object of the present paper is to determine coefficient estimates, distortion bounds, closure theorems and extreme points for functions $f(z)$ belonging to a new subclass of uniformly starlike functions.
\end{abstract}

Keywords: Univalent, Uniformly starlike function, Hypergeometric function.

\section{Introduction}

Let $\mathrm{A}$ denote the class of functions $f(z)$ of the form

$$
f(z)=z+\sum_{n=2}^{\infty} a_{n} z^{n}
$$

which are analytic in the open unit disk $\mathrm{Y}=\{z \in \mathrm{X}:|z|<1\}$. Further, by $\Sigma$ we shall denote the class of functions $f \in \mathrm{A}$ which are univalent in $\mathrm{Y}$

For $f \in \mathrm{A}$ given by (1) and $g(z)$ given by

$$
g(z)=z+\sum_{n=2}^{\infty} b_{n} z^{n}
$$

their convolution (or Hadamard product), denoted by $(f * g)$, is defined as

$$
(f * g)(z)=z+\sum_{n=2}^{\infty} a_{n} b_{n} z^{n}, \quad(z \in \mathrm{Y}) .
$$

Note that $f * g \in \mathrm{A}$.

A function $f \in \mathrm{A}$ is said to be in $\beta-U S(\alpha)$, the class of $\beta$ - uniformly starlike functions of order $\alpha, 0 \leq \alpha<1$, if satisfies the condition

$$
\operatorname{Re}\left\{\frac{z f^{\prime}(z)}{f(z)}\right\}>\beta\left|\frac{z f^{\prime}(z)}{f(z)}-1\right|+\alpha \quad(\beta \geq 0),
$$

and a function $f \in \mathrm{A}$ is said to be in $\beta-U C(\alpha)$, the class of $\beta$ - uniformly convex functions of order $\alpha$, $0 \leq \alpha<1$, if satisfies the condition

$$
\operatorname{Re}\left\{1+\frac{z f^{\prime \prime}(z)}{f^{\prime}(z)}\right\}>\beta\left|\frac{z f^{\prime \prime}(z)}{f^{\prime}(z)}\right|+\alpha \quad(\beta \geq 0) .
$$

Uniformly starlike and uniformly convex functions were first introduced by $[1,2]$ and then studied by various authors $[3$, 4]. It is known that $\beta-U S(\alpha)$ or $\beta-U C(\alpha)$ if and only if $1+\frac{z f^{\prime \prime}(z)}{f^{\prime}(z)}$ or $\frac{z f^{\prime}(z)}{f(z)}$, respectively, takes all the values in the conic domain $R_{\beta, \alpha}$ which is included in the right half plane given by

$$
R_{\beta, \alpha}=\omega=u+i v \in C: u>\beta \sqrt{(u-1)^{2}+v^{2}}+\alpha,
$$

$\beta \geq 0$ and $0 \leq \alpha<1$

Denote by $P_{\beta, \alpha}(\beta \geq 0,0 \leq \alpha<1)$ of functions $\mathrm{p}$, such that $p \in P_{\beta, \alpha}$, where $\mathrm{P}$ denotes well known class of Caratheodory functions. The function $P_{\beta, \alpha}$ maps the unit disk conformally onto the domain $R_{\beta, \alpha}$ such that $1 \in R_{\beta, \alpha}$ and $\partial R_{\beta, \alpha}$ is a curve defined by the equality $\partial R_{\beta, \alpha}=\omega=u+i v \in C: u^{2}=\left(\beta \sqrt{(u-1)^{2}+v^{2}}+\alpha\right)^{2}$, $\beta \geq 0$ and $0 \leq \alpha<1$.

From elementary computations we see that (7) represents conic sections symmetric about the real axis. Thus $R_{\beta, \alpha}$ is an elliptic domain for $\beta>1$, a parabolic domain for $\beta=1$, a hyperbolic domain for $0<\beta<1$ and the right half plane $u>\alpha$, for $\beta=0$.

In [5], Sakaguchi (1959) defined the class $S_{s}$ of starlike functions with respect to symmetric points as follows:

Let $f \in \mathrm{A}$. Then $f$ is said to be starlike with respect to symmetric points in $\mathrm{Y}$ if and only if

$$
\operatorname{Re}\left\{\frac{2 z f^{\prime}(z)}{f(z)-f(-z)}\right\}>0 \quad(z \in \mathrm{Y}) .
$$

Recently, Owa et. al. (2007) [6] defined and studied the class $S_{s}(\alpha, t)$, 


\section{International Journal of Science and Research (IJSR) \\ ISSN (Online): 2319-7064}

Index Copernicus Value (2016): 6.14 | Impact Factor (2015): 6.391

$$
\operatorname{Re}\left\{\frac{(1-t) z f^{\prime}(z)}{f(z)-f(t z)}\right\}>\alpha \quad(z \in U),
$$

where $0 \leq \alpha<1,|t| \leq 1, t \neq 1$.

In 2008, Selvaraj and Karthikeyan [7] defined the following operator $D_{\lambda}^{m}\left(\alpha_{1}, \beta_{1}\right) f: \mathrm{Y} \rightarrow \mathrm{Y}$ by

$D_{\lambda}^{0}\left(\alpha_{1} ; \beta_{1}\right) f(z)=f(z) * G_{q, s}\left(\alpha_{1}, \beta_{1} ; z\right)$,

$D_{\lambda}^{1}\left(\alpha_{1} ; \beta_{1}\right) f(z)=(1-\lambda)\left(f(z) * G_{q, s}\left(\alpha_{1}, \beta_{1} ; z\right)\right)$

$$
+\lambda z\left(f(z) * G_{q, s}\left(\alpha_{1}, \beta_{1} ; z\right)\right)^{\prime}
$$

$D_{\lambda}^{m}\left(\alpha_{1} ; \beta_{1}\right) f(z)=D_{\lambda}^{1}\left(D_{\lambda}^{m-1}\left(\alpha_{1} ; \beta_{1}\right) f(z)\right)$,

where $m \in \mathrm{N}_{0}=\mathrm{N} \cup\{0\}$ and $\lambda \geq 0$.

If $f \in \mathrm{A}$, then from (10) we may easily deduce that

$D_{\lambda}^{m}\left(\alpha_{1} ; \beta_{1}\right) f(z)$

$=z+\sum_{n=2}^{\infty}[1+(n-1) \lambda]^{m} \Gamma_{n}\left(\alpha_{1}\right) a_{n} z^{n}$

where $\Gamma_{n}\left(\alpha_{1}\right)=\frac{\left(\alpha_{1}\right)_{n-1} \ldots\left(\alpha_{q}\right)_{n-1}}{\left(\beta_{1}\right)_{n-1} \ldots\left(\beta_{s}\right)_{n-1}} \frac{1}{(n-1) !}$.

Special cases of the operator $D_{\lambda}^{m}\left(\alpha_{1} ; \beta_{1}\right) f$ includes various other linear operators which were considered in many earlier work on the subject of analytic and univalent functions. If we let $m=0$ in $D_{\lambda}^{m}\left(\alpha_{1} ; \beta_{1}\right) f$, we have

$$
D_{\lambda}^{0}\left(\alpha_{1} ; \beta_{1}\right) f(z)=\mathrm{H}_{q}^{1}\left(\alpha_{1} ; \beta_{1}\right) f(z)
$$

where $\mathrm{H}_{q, s}^{1}\left(\alpha_{1} ; \beta_{1}\right)$ is Dziok-Srivastava operator for functions in A (see [8]) and for $q=2, s=1, \alpha_{1}=\beta_{1}, \alpha_{2}=1$ and $\lambda=1$, we get the operator introduced by Salagean (1983)([9]).

Definition 1.1 A function $f(z) \in \mathrm{A}$ is said to be in the class $k-\mathrm{Y} \Sigma_{s}\left(\lambda, \alpha_{1}, \beta_{1}, \gamma, t\right)$ iffor all $z \in \mathrm{Y}$,

$$
\begin{gathered}
\operatorname{Re}\left\lfloor\frac{(1-t) z\left(D_{\lambda}^{m}\left(\alpha_{1}, \beta_{1}\right) f(z)\right)}{D_{\lambda}^{m}\left(\alpha_{1}, \beta_{1}\right) f(z)-D_{\lambda}^{m}\left(\alpha_{1}, \beta_{1}\right) f(t z)}-\gamma \mid\right. \\
\quad \geq k\left|\frac{(1-t) z\left(D_{\lambda}^{m}\left(\alpha_{1}, \beta_{1}\right) f(z)\right)^{\prime}}{D_{\lambda}^{m}\left(\alpha_{1}, \beta_{1}\right) f(z)-D_{\lambda}^{m}\left(\alpha_{1}, \beta_{1}\right) f(t z)}\right|
\end{gathered}
$$

for $\lambda \geq 0, k, m \geq 0,|t| \leq 1, t \neq 1$ and $0 \leq \gamma<1$.

Furthermore, we say that a function $f(z) \in k-\mathrm{Y} \Sigma_{s}\left(\lambda, \alpha_{1}, \beta_{1}, \gamma, t\right)$ is in the subclass $k-\mathrm{Y} \Sigma \mathrm{T}_{s}\left(\lambda, \alpha_{1}, \beta_{1}, \gamma, t\right)$ if $f(z)$ is of the following form: $f(z)=z-\sum_{n=2}^{\infty} a_{n} z^{n} \quad\left(a_{n} \geq 0, n \in N\right)$.

The main objective of this paper is to study the coefficient estimates, extreme points, distortion bounds and closure properties for $f(z) \in k-\mathrm{Y} \Sigma \mathrm{T}_{s}\left(\lambda, \alpha_{1}, \beta_{1}, \gamma, t\right)$ by fixing second coefficients.
Similar other classes of univalent functions with fixed second coefficients have been extensively studied by Aouf (1997)[10, 11], S. M. Khairnar et. al., (2010), [12], Darwish (2008)[13], and others see [14].

\section{Coefficient Estimate}

Lemma 2.1 Let $\omega=u+i v$. Then Re $\omega \geq \alpha$ if and only if $|\omega-(1+\alpha)| \leq|\omega+(1+\alpha)|$

Lemma 2.2 Let $\omega=u+i v$ and $\alpha, \gamma$ are real numbers.

Then $\quad \operatorname{Re} \omega>\alpha|\omega-1|+\gamma \quad$ if and only if $\operatorname{Re}\left\{\omega\left(1+\alpha e^{i \theta}\right)-\alpha e^{i \theta}\right\}>\gamma$.

Theorem 2.3 Let the function $f(z)$ be defined by (13). Then $f(z) \in k-\mathrm{Y} \Sigma \mathrm{T}_{s}\left(\lambda, \alpha_{1}, \beta_{1}, \gamma, t\right)$ if and only if

$$
\sum_{n=2}^{\infty} C_{n}(m, \lambda, k, \gamma) a_{n} \leq(1-\gamma)
$$

where

$$
C_{n}(m, \lambda, k, \gamma)
$$

$$
=(1+(n-1) \lambda)^{m} \Gamma_{n}\left(\alpha_{1}\right)\left[n(k+1)-(k+\gamma) u_{n}\right]^{\prime}
$$

$\lambda \geq 0, \quad k, m \geq 0, \quad 0 \leq \gamma<1, \quad|t| \leq 1, \quad t \neq 1 \quad$ and $u_{n}=1+t+t^{2}+\cdots+t^{n}$.

The result is sharp for the function $f(z)$ is given by

$$
f(z)=z-\frac{1-\gamma}{C_{n}(m, \lambda, k, \gamma)} z^{n} .
$$

Proof. By definition $f(z) \in k-\mathrm{Y} \Sigma \mathrm{T}_{s}\left(\lambda, \alpha_{1}, \beta_{1}, \gamma, t\right)$ if and only if the condition (12) is satisfied.

Then by Lemma 2.1, we have

$$
\begin{aligned}
& \operatorname{Re}\left\lfloor\frac{(1-t) z\left(D_{\lambda}^{m}\left(\alpha_{1}, \beta_{1}\right) f(z)\right)^{\prime}}{D_{\lambda}^{m}\left(\alpha_{1}, \beta_{1}\right) f(z)-D_{\lambda}^{m}\left(\alpha_{1}, \beta_{1}\right) f(t z)}\left(1+k e^{i \theta}\right)-k e^{i \theta}\right\rfloor \\
& \quad \geq \gamma, \quad-\pi<\theta \leq \pi .
\end{aligned}
$$

It is also written as

$$
\begin{aligned}
& \quad \operatorname{Re}\left\{\frac{(1-t) z\left(D_{\lambda}^{m}\left(\alpha_{1}, \beta_{1}\right) f(z)\right)^{\prime}\left(1+k e^{i \theta}\right)}{D_{\lambda}^{m}\left(\alpha_{1}, \beta_{1}\right) f(z)-D_{\lambda}^{m}\left(\alpha_{1}, \beta_{1}\right) f(t z)}\right\} \\
& -\frac{k e^{i \theta} D_{\lambda}^{m}\left(\alpha_{1}, \beta_{1}\right) f(z)-D_{\lambda}^{m}\left(\alpha_{1}, \beta_{1}\right) f(t z)}{D_{\lambda}^{m}\left(\alpha_{1}, \beta_{1}\right) f(z)-D_{\lambda}^{m}\left(\alpha_{1}, \beta_{1}\right) f(t z)} \geq \gamma . \\
& \text { Let } A(z)=(1-t) z\left(D_{\lambda}^{m}\left(\alpha_{1}, \beta_{1}\right) f(z)\right)^{\prime}\left(1+k e^{i \theta}\right) \\
& -k e^{i \theta}\left(D_{\lambda}^{m}\left(\alpha_{1}, \beta_{1}\right) f(z)-D_{\lambda}^{m}\left(\alpha_{1}, \beta_{1}\right) f(t z)\right) \\
& \text { and } B(z)=D_{\lambda}^{m}\left(\alpha_{1}, \beta_{1}\right) f(z)-D_{\lambda}^{m}\left(\alpha_{1}, \beta_{1}\right) f(t z) .
\end{aligned}
$$

From Lemma 2.1 and Lemma 2.2,

$|A(z)+(1-\gamma) B(z)| \geq|A(z)-(1+\gamma) B(z)|$ for $\quad 0 \leq \gamma<1$.

Using a simple computation,

$|A(z)+(1-\gamma) B(z)| \geq$ 


\section{International Journal of Science and Research (IJSR) \\ ISSN (Online): 2319-7064}

Index Copernicus Value (2016): 6.14 | Impact Factor (2015): 6.391

$$
\begin{aligned}
& |1-t|\left\{(2-\gamma)|z|-\sum_{n=2}^{\infty}(1+(n-1) \lambda)^{m} \Gamma_{n}\left(\alpha_{1}\right)\left[n+(1-\gamma) u_{n}\right] a_{n}|z|^{n}\right. \\
& \left.-k \sum_{n=2}^{\infty}(1+(n-1) \lambda)^{m} \Gamma_{n}\left(\alpha_{1}\right)\left[n-u_{n}\right] a_{n}|z|^{n}\right\} . \\
& \text { Also, }|A(z)-(1+\gamma) B(z)| \leq \\
& |1-t|\left\{\gamma|z|+\sum_{n=2}^{\infty}(1+(n-1) \lambda)^{m} \Gamma_{n}\left(\alpha_{1}\right)\left[n-(1+\gamma) u_{n}\right] a_{n}|z|^{n}\right. \\
& \left.+k \sum_{n=2}^{\infty}(1+(n-1) \lambda)^{m} \Gamma_{n}\left(\alpha_{1}\right)\left[n-u_{n}\right] a_{n}|z|^{n}\right\} \text {. }
\end{aligned}
$$$$
\text { Now } \quad|A(z)+(1-\gamma) B(z)|-|A(z)-(1+\gamma) B(z)|
$$$$
\geq\left\{2(1-\gamma)|z|-2 \sum_{n=2}^{\infty}(1+(n-1) \lambda)^{m} \Gamma_{n}\left(\alpha_{1}\right)\right.
$$$$
\left.\left[n(k+1)-(k+\gamma) u_{n}\right] a_{n}|z|^{n}\right\}
$$

$$
\begin{aligned}
a_{n} & \leq \frac{(1-\gamma)}{(1+(n-1) \lambda)^{m} \Gamma_{n}\left(\alpha_{1}\right)\left[n(k+1)-(k+\gamma) u_{n}\right]} \\
& \leq \frac{(1-\gamma)}{C_{n}(m, \lambda, k, \gamma)} \quad(n \geq 2) .
\end{aligned}
$$

where

$C_{n}(m, \lambda, k, \gamma)=(1+(n-1) \lambda)^{m} \Gamma_{n}\left(\alpha_{1}\right)\left[n(k+1)-(k+\gamma) u_{n}\right]$ $, \lambda \geq 0, k, m \geq 0$ and $0 \leq \gamma<1$.

Setting $n=2$ in (16), we have

$$
\begin{aligned}
a_{2} & \leq \frac{(1-\gamma)\left(\beta_{1}\right)}{(1+\lambda)^{m}[(2+k-\gamma)-t(\gamma+k)]\left(\alpha_{1}\right)} \\
& \leq \frac{(1-\gamma)\left(\beta_{1}\right)}{C_{2}(m, \lambda, k, \gamma)\left(\alpha_{1}\right)} .
\end{aligned}
$$

where $C_{2}(m, \lambda, k, \gamma)=(1+\lambda)^{m}[(2+k-\gamma)-t(\gamma+k)]$. Let $k-\mathrm{Y} \Sigma \mathrm{T}_{s}\left(c, \lambda, \alpha_{1}, \beta_{1}, \gamma, t\right)$ denote the class of functions $f(z)$ in $k-\mathrm{Y} \sum \mathrm{T}_{s}\left(\lambda, \alpha_{1}, \beta_{1}, \gamma, t\right)$ of the form

$$
\begin{gathered}
f(z)=z-\frac{c(1-\gamma)\left(\beta_{1}\right)}{C_{2}(m, \lambda, k, \gamma)\left(\alpha_{1}\right)} z^{2}-\sum_{n=3}^{\infty} a_{n} z^{n} \\
\left(a_{n} \geq 0\right), 0 \leq c \leq 1 .
\end{gathered}
$$

$$
\leq(1-\gamma) \text {. }
$$

which gives the desired estimation.

Conversely, suppose that (14) holds. Then we must show that

$$
\operatorname{Re}\left\{\frac{(1-t) z\left(D_{\lambda}^{m}\left(\alpha_{1}, \beta_{1}\right) f(z)\right)^{\prime}\left(1+k e^{i \theta}\right)-k e^{i \theta}\left[D_{\lambda}^{m}\left(\alpha_{1}, \beta_{1}\right) f(z)-D_{\lambda}^{m}\left(\alpha_{1}, \beta_{1}\right) f(t z)\right]}{D_{\lambda}^{m}\left(\alpha_{1}, \beta_{1}\right) f(z)-D_{\lambda}^{m}\left(\alpha_{1}, \beta_{1}\right) f(t z)}\right\} \geq \gamma \text {. }
$$

Remark 2.1 If $\alpha_{1}=\beta_{1}=1$, then the result is reduced to the class $k-\tilde{\mathrm{Y}} \Sigma_{s}(\lambda, \mu, \gamma, t)$ studied by Murat Caglar and Orhan [15]. Take $t=0, \beta=0$ and $\lambda=1$, this result is reduced into the class $T(n, \alpha)$ studied by Aouf [10]. If Choosing the values of $z$ on the positive real axis where $0 \leq z=r<1$, the above inequality reduces to

$$
\operatorname{Re}\left\{\frac{(1-\gamma)-\sum_{n=2}^{\infty}(1+(n-1) \lambda)^{m} \Gamma_{n}\left(\alpha_{1}\right)\left(n\left(1+k e^{i \theta}\right)-u_{n}\left(\gamma+k e^{i \theta}\right)\right) a_{n} z^{n-1}}{1-\sum_{n=2}^{\infty}(1+(n-1) \lambda)^{m} \Gamma_{n}\left(\alpha_{1}\right) u_{n} a_{n} z^{n-1} / p / n}\right\}
$$

$\geq 0$. $t=0, \beta=0, \alpha_{1}=\beta_{1}=1$ and $\lambda=1$, then the result was reduced into the class $\operatorname{UCT}(\alpha, \beta)$ by Khairanar (2010)[12].

Theorem 2.5 Let the function $f(z)$ be defined by (18). Then $f(z)$ in $k-\mathrm{Y} \mathrm{T}_{s}\left(c, \lambda, \alpha_{1}, \beta_{1}, \gamma, t\right)$ if and only if

$$
\sum_{n=3}^{\infty} C_{n}(m, \lambda, k, \gamma) a_{n} \leq(1-\gamma)(1-c) \text {. }
$$

Since $\operatorname{Re}\left(-e^{i \theta}\right) \geq-\left|e^{i \theta}\right|=-1$, the above inequality Proof. reduces to

$$
\operatorname{Re}\left\{\frac{(1-\gamma)-\sum_{n=2}^{\infty}(1+(n-1) \lambda)^{m} \Gamma_{n}\left(\alpha_{1}\right)\left(n(1+k)-(\gamma+k) u_{n}\right) a_{n} r^{n-1}}{1-\sum_{n=2}^{\infty}(1+(n-1) \lambda)^{m} \Gamma_{n}\left(\alpha_{1}\right) u_{n} a_{n} r^{n-1}}\right\}
$$

$\geq 0$.

Letting $r \rightarrow 1^{-}$, we have desired conclusion.

$$
\begin{aligned}
& a_{2}=\frac{c(1-\gamma)\left(\beta_{1}\right)}{C_{2}(m, \lambda, k, \gamma)\left(\alpha_{1}\right)}, \quad 0 \leq c \leq 1 \text {, in } \\
& \text { implifying we get the result. } \\
& \text { Corollary } 2.6 \text { Let the function } f(z) \text { defined by }(18) \\
& \quad \text { class } \quad k-\mathrm{Y} \mathrm{T}_{s}\left(c, \lambda, \alpha_{1}, \beta_{1}, \gamma, t\right) \text {, } \\
& a_{n} \leq \frac{(1-\gamma)(1-c)}{C_{n}(m, \lambda, k, \gamma)} \quad(n \geq 3) \quad 0 \leq c \leq 1 .
\end{aligned}
$$

Substituting

Corollary 2.4 Let the function $f(z)$ defined by (13) be in the class $k-\mathrm{Y} \Sigma \mathrm{T}_{s}\left(\lambda, \alpha_{1}, \beta_{1}, \gamma, t\right)$, then 


\section{International Journal of Science and Research (IJSR) \\ ISSN (Online): 2319-7064}

Index Copernicus Value (2016): 6.14 | Impact Factor (2015): 6.391

\section{Extreme Points}

Theorem 3.1 Let

$f_{2}(z)=z-\frac{c(1-\gamma)\left(\beta_{1}\right)}{C_{2}(c, m, \lambda, k, \gamma)\left(\alpha_{1}\right)} z^{2}$

and

$f_{n}(z)=z-\frac{c(1-\gamma)\left(\beta_{1}\right)}{C_{2}(c, m, \lambda, k, \gamma)\left(\alpha_{1}\right)} z^{2}-\frac{(1-\gamma)(1-c)}{C_{n}(m, \lambda, k, \gamma)} z^{n}$

for $n=3,4, \cdots$. Then $f(z)$ is in the class $k-\mathrm{Y} \sum \mathrm{T}_{s}\left(c, \lambda, \alpha_{1}, \beta_{1}, \gamma, t\right)$ if and only if it can be expressed in the form $f(z)=\sum_{n=2}^{\infty} \mu_{n} f_{n}(z)$, where $\mu_{n} \geq 0$ and $\sum_{n=2}^{\infty} \mu_{n}=1$.

Proof. We suppose that $f(z)$ can be expressed in the form (23). Then we have

$f_{n}(z)=z-\frac{c(1-\gamma)\left(\beta_{1}\right)}{C_{2}(c, m, \lambda, k, \gamma)\left(\alpha_{1}\right)} z^{2}-\frac{(1-\gamma)(1-c) \mu_{n}}{C_{n}(m, \lambda, k, \gamma)} z^{n} .+\left(\frac{2 c^{2}(1-\gamma)^{2} \beta_{1}^{2}}{\left(C_{2}(m, \lambda, k, \gamma)\right)^{2} \alpha_{1}^{2}}+\frac{2(1-c)(1-\gamma)\left(\beta_{1}\right)}{\left(C_{3}(m, \lambda, k, \gamma)\right)^{2}}\right) r^{2}$

Since $\sum_{n=3}^{\infty} \frac{(1-\gamma)(1-c) \mu_{n}}{C_{n}(m, \lambda, k, \gamma)} \frac{C_{n}(m, \lambda, k, \gamma)}{(1-\gamma)}$ $\leq(1-c)\left(1-\mu_{2}\right)$ $\leq(1-\gamma)$

it follows from (14) that $f(z)$ is in the class $k-\mathrm{Y} \Sigma \mathrm{T}_{s}\left(c, \lambda, \alpha_{1}, \beta_{1}, \gamma\right)$. Conversely, suppose that $f(z)$ defined by (18) is in the class $k-\mathrm{Y} \mathrm{T}_{s}\left(c, \lambda, \alpha_{1}, \beta_{1}, \gamma\right)$. Then, by using (20), we get

$$
a_{n} \leq \frac{(1-c)(1-\gamma)}{C_{n}(m, \lambda, k, \gamma)} \quad(n \geq 3) \text {. }
$$

Setting

$$
\mu_{n}=\frac{C_{n}(m, \lambda, k, \gamma)}{(1-c)(1-\gamma)} a_{n} \quad(n \geq 3)
$$

and $\mu_{2}=1-\sum_{n=3}^{\infty} \mu_{n},(28)$

we have (23). This completes the proof of the theorem.

Corollary 3.2 The extreme points of the class $k-{\mathrm{Y} \Sigma \mathrm{T}_{s}}_{s}\left(c, \lambda, \alpha_{1}, \beta_{1}, \gamma, t\right)$ are functions $f_{n}(z), n \geq 2$ given by (3.1).

\section{Growth and Distortion Theorem}

The following lemmas are required in our investigation of growth and distortion properties of the general class $k-\mathrm{Y} \mathrm{T}_{s}\left(c, \lambda, \alpha_{1}, \beta_{1}, \gamma, t\right)$.

where and Silvia (1981)([16]), since

$$
\frac{\partial\left|f_{3}\left(r e^{i \theta}\right)\right|^{2}}{\partial \theta}=2(1-\gamma) r^{3} \sin \theta \times
$$

Lemma 4.1 Let the function $f_{3}(z)$ be defined by

$$
f_{3}(z)=z-\frac{c(1-\gamma)\left(\beta_{1}\right)}{C_{2}(m, \lambda, k, \gamma)(\alpha 1)} z^{2}-\frac{(1-\gamma)(1-c)}{C_{3}(m, \lambda, k, \gamma)} z^{3},
$$

For some $\mathrm{n}$. Then for $0 \leq r<1$ and $0 \leq c \leq 1$,

$$
\left|f_{3}\left(r e^{i \theta}\right)\right| \geq r-\frac{c(1-\gamma)\left(\beta_{1}\right)}{C_{2}(m, \lambda, k, \gamma)\left(\alpha_{1}\right)} r^{2}-\frac{(1-\gamma)(1-c)}{C_{3}(m, \lambda, k, \gamma)} r^{3}
$$

with equality for $\theta=0$. For either $0 \leq c<c_{0}$ and $0 \leq r \leq r_{0}$ or $c_{0} \leq c \leq 1$,

$$
\left|f_{3}\left(r e^{i \theta}\right)\right| \leq r+\frac{c(1-\gamma)\left(\beta_{1}\right)}{C_{2}(m, \lambda, k, \gamma)\left(\alpha_{1}\right)} r^{2}-\frac{(1-\gamma)(1-c)}{C_{3}(m, \lambda, k, \gamma)} r^{3}
$$

with equality for $\theta=\pi$. Further, for $0 \leq c \leq c_{0}$ and $r_{0} \leq r<1$,
$\left.+\left(\frac{(1-c)^{2}(1-\gamma)^{2}}{\left(C_{3}(m, \lambda, k, \gamma)\right)^{2}}+\frac{c^{2}(1-c)(1-\gamma)^{3}\left(\beta_{1}^{2}\right)}{2\left(C_{2}(m, \lambda, k, \gamma)\right)^{2}\left(C_{3}(m, \lambda, k, \gamma) \alpha_{1}^{2}\right)}\right) r^{4}\right\}^{\frac{1}{2}}$

with equality for

$\theta=\cos ^{-1}\left(\frac{c(1-c)(1-\gamma)\left(\beta_{1}\right) r^{2}-c\left(C_{3}(m, \lambda, k, \gamma)\right)\left(\beta_{1}\right)}{4(1-c)\left(C_{2}(m, \lambda, k, \gamma)\right)\left(\alpha_{1}\right) r}\right)$

$$
\begin{gathered}
c_{0}=\frac{\left[(1-\gamma)\left(\beta_{1}\right)-4\left(C_{2}(m, \lambda, k, \gamma)\right)\left(\alpha_{1}\right)-C_{3}(m, \lambda, k, \gamma)\left(\beta_{1}\right)\right]+c_{0}^{*}}{2(1-\gamma)\left(\beta_{1}\right)} \\
c_{0}^{*}=\left\{\left[(1-\gamma)\left(\beta_{1}\right)-4\left(C_{2}(m, \lambda, k, \gamma)\right)\left(\alpha_{1}\right)-C_{3}(m, \lambda, k, \gamma)\left(\beta_{1}\right)\right]^{2}\right. \\
\left.+16 C_{2}(m, \lambda, k, \gamma)(1-\gamma)\left(\alpha_{1}\right)\left(\beta_{1}\right)\right\}^{\frac{1}{2}}
\end{gathered}
$$

$$
\begin{aligned}
& \text { and } r_{0}=\frac{-2(1-c)\left(C_{2}(m, \lambda, k, \gamma)\left(\alpha_{1}\right)\right)+r_{0}^{*}}{c(1-c)(1-\gamma)\left(\beta_{1}\right)} \\
& * * r_{0}^{*}=\left\{4(1-c)^{2}\left(C_{2}(m, \lambda, k, \gamma)\right)^{2}\left(\alpha_{1}^{2}\right)+c^{2}(1-c)(1-\gamma) C_{3}(m, \lambda, k, \gamma)\left(\beta_{1}^{2}\right)\right\}^{\frac{1}{2}}
\end{aligned}
$$

Proof. We employ the same technique as used by Silverman 


\section{International Journal of Science and Research (IJSR) \\ ISSN (Online): 2319-7064}

Index Copernicus Value (2016): 6.14 $\mid$ Impact Factor (2015): 6.391

$\left|\frac{c(\beta 1)}{C_{2}(m, \lambda, k, \gamma)(\alpha 1)}-\frac{c(1-c)(1-\gamma)(\beta 1)}{C_{2}(m, \lambda, k, \gamma) C_{3}(m, \lambda, k, \gamma)(\alpha 1)} r^{2}+\frac{4(1-c)}{C_{3}(m, \lambda, k, \gamma)} r \cos \theta\right|$

we can see that

$$
\frac{\partial\left|f_{3}\left(r e^{i \theta}\right)\right|^{2}}{\partial \theta}=0
$$

for $\theta_{1}=0, \theta_{2}=\pi$ and

$$
\theta_{3}=\cos ^{-1}\left(\frac{c(1-c)(1-\gamma)\left(\beta_{1}\right) r^{2}-c\left(\beta_{1}\right) C_{3}(m, \lambda, k, \gamma)}{4(1-c) C_{2}(m, \lambda, k, \gamma)\left(\alpha_{1}\right) r}\right)
$$

Since $\theta_{3}$ is a valid root only when $-1 \leq \cos \theta_{3} \leq 1$, we have a third root if and only if $r_{0} \leq r<1$ and $0 \leq c<c_{0}$. Thus the results of Lemma 4.1 follow upon comparing the extremal values $\left|f_{3}\left(r e^{i \theta_{k}}\right)\right|,(k=1,2,3)$ on the appropriate intervals.

Lemma 4.2 Let the function $f_{n}(z)(n \geq 4)$ be defined by (22). Then

$$
\left|f_{n}\left(r e^{i \theta}\right) \leq\right| f_{4}(-r) \mid \quad(n \geq 4) .
$$

Proof. Since

$f_{n}(z)=z-\frac{c(1-\gamma)\left(\beta_{1}\right)}{C_{2}(c, m, \lambda, k, \gamma)\left(\alpha_{1}\right)} z^{2}-\frac{(1-\gamma)(1-c)}{C_{n}(m, \lambda, k, \gamma)} z^{n}$ and $\frac{(1-\gamma)(1-c)}{C_{n}(m, \lambda, k, \gamma)} r^{n}$ is a decreasing function of $n$, we have $\left|f_{n}\left(r e^{i \theta}\right)\right| \leq r+\frac{c(1-\gamma)\left(\beta_{1}\right)}{C_{2}(c, m, \lambda, k, \gamma)\left(\alpha_{1}\right)} r^{2}$

$$
+\frac{(1-\gamma)(1-c)}{C_{n}(m, \lambda, k, \gamma)} r^{4}=-f_{4}(-r)
$$

which proves (38).

Theorem 4.3 Let the function $f(z)$ defined by (18) be in the class $k-\mathrm{Y} \sum \mathrm{T}_{s}\left(c, \lambda, \alpha_{1}, \beta_{1}, \gamma, t\right)$. Then, for $0 \leq r<1$,

$\left|f\left(r e^{i \theta}\right)\right| \geq r-\frac{c(1-\gamma)\left(\beta_{1}\right)}{C_{2}(m, \lambda, k, \gamma)\left(\alpha_{1}\right)} r^{2}-\frac{(1-c)(1-\gamma)}{C_{3}(m, \lambda, k, \gamma)} r^{3}$

with equality for $f_{3}(z)$ at $z=r$ and $\left|f\left(r e^{i \theta}\right)\right| \leq \max \left\{\max _{\theta}\left|f_{3}\left(r e^{i \theta}\right)\right|,-f_{4}(-r)\right\}$

where $\max _{\theta}\left|f_{3}\left(r e^{i \theta}\right)\right|$ is given by Lemma 4.1.

The proof of this theorem is obtained by comparing the bounds given by Lemma 4.1 and Lemma 4.2. Putting $c=1$ in theorem 4.3 , we obtain the following corollary.

Corollary 4.4 Let the function $f(z)$ defined by (13) be in the class $k-\mathrm{Y} \Sigma \mathrm{T}_{s}\left(c, \lambda, \alpha_{1}, \beta_{1}, \gamma, t\right)$. Then, for $0 \leq r<1$,
$r-\frac{(1-\gamma)\left(\beta_{1}\right)}{C_{2}(m, \lambda, k, \gamma)\left(\alpha_{1}\right)} r^{2} \leq|f(z)| \leq r+\frac{(1-\gamma)\left(\beta_{1}\right)}{C_{2}(m, \lambda, k, \gamma)\left(\alpha_{1}\right)} r^{2}$.

(41)

The result is sharp for the function

$$
f(z)=z-\frac{(1-\gamma)\left(\beta_{1}\right)}{C_{2}(m, \lambda, k, \gamma)\left(\alpha_{1}\right)} z^{2} .
$$

Putting $c=1$ and $k=0$ in theorem 4.3, we obtain the following corollary.

Corollary 4.5 Let the function $f(z)$ defined by (13) be in the class $k-\mathrm{Y} \sum \mathrm{T}_{s}\left(c, \lambda, \alpha_{1}, \beta_{1}, \gamma, t\right)$. Then, for $0 \leq r<1$,

$r-\frac{(1-\gamma)\left(\beta_{1}\right)}{C_{2}(m, \lambda, 0, \gamma)\left(\alpha_{1}\right)} r^{2} \leq|f(z)| \leq r+\frac{(1-\gamma)\left(\beta_{1}\right)}{C_{2}(m, \lambda, 0, \gamma)\left(\alpha_{1}\right)} r^{2}$

The result is sharp for the function

$$
f(z)=z-\frac{(1-\gamma)\left(\beta_{1}\right)}{C_{2}(m, \lambda, k, \gamma)\left(\alpha_{1}\right)} z^{2} .
$$

Remark 4.1 If $\alpha_{1}=\beta_{1}=1, m=1$ and $t=-1$ then the above result (corollary 4.4) is reduced to the class $S_{s}(\lambda, k, \beta)$ studied by C.Selvaraj et.al. (2009)[17],

$$
r-\frac{(1-\gamma)}{2(1+k)(1+\lambda)} r^{2} \leq|f(z)| \leq r+\frac{(1-\gamma)\left(\beta_{1}\right)}{2(1+k)(1+\lambda)} r^{2}
$$

Lemma 4.6 Let the function $f_{3}(z)$ be defined by (29).

Then, for $0 \leq r<1$ and $0 \leq c \leq 1$,

$$
\left|f_{3}^{\prime}\left(r e^{i \theta}\right)\right| \geq 1-\frac{2 c(1-\gamma)\left(\beta_{1}\right)}{C_{2}(m, \lambda, k, \gamma)\left(\alpha_{1}\right)} r-\frac{3(1-\gamma)(1-c)}{C_{3}(m, \lambda, k, \gamma)} r^{2}
$$

with equality for $\theta=0$. For either $0 \leq c<c_{1}$ and $r_{1} \leq r \leq 1$

$$
\left|f_{3}^{\prime}\left(r e^{i \theta}\right)\right| \leq 1+\frac{2 c(1-\gamma)\left(\beta_{1}\right)}{C_{2}(m, \lambda, k, \gamma)\left(\alpha_{1}\right)} r-\frac{3(1-\gamma)(1-c)}{C_{3}(m, \lambda, k, \gamma)} r^{3}
$$

with equality for $\theta=\pi$. Furthermore, for $0 \leq c \leq c_{1}$ and $r_{1} \leq r<1$

$$
\begin{aligned}
\left|f_{3}^{\prime}\left(r e^{i \theta}\right)\right| & \leq\left\{\left(1+\frac{c^{2}(1-\gamma) \beta_{1}^{2} C_{3}(m, \lambda, k, \gamma)}{3(1-c)\left(C_{2}(m, \lambda, k, \gamma)\right)^{2} \alpha_{1}^{2}}\right)\right. \\
& +\left(\frac{4 c^{2}(1-\gamma)^{2} \beta_{1}^{2}}{\left(C_{2}(m, \lambda, k, \gamma)\right)^{2} \alpha_{1}^{2}}+\frac{6(1-c)(1-\gamma)\left(\beta_{1}\right)}{\left(C_{3}(m, \lambda, k, \gamma)\right)^{2}}\right) r^{2} \\
& \left.+\left(\frac{9(1-c)^{2}(1-\gamma)^{2}}{\left(C_{3}(m, \lambda, k, \gamma)\right)^{2}}+\frac{6 c^{2}(1-c)(1-\gamma)^{3}\left(\beta_{1}^{2}\right)}{2\left(C_{2}(m, \lambda, k, \gamma)\right)^{2}\left(C_{3}(m, \lambda, k, \gamma) \alpha_{1}^{2}\right)}\right) r^{4}\right\}^{\frac{1}{2}}
\end{aligned}
$$




\section{International Journal of Science and Research (IJSR) \\ ISSN (Online): 2319-7064}

Index Copernicus Value (2016): 6.14 | Impact Factor (2015): 6.391

with equality for

$$
\theta=\cos ^{-1}\left(\frac{3 c(1-c)(1-\gamma)\left(\beta_{1}\right) r^{2}-c\left(C_{3}(m, \lambda, k, \gamma)\right)\left(\beta_{1}\right)}{6(1-c)\left(C_{2}(m, \lambda, k, \gamma)\right)\left(\alpha_{1}\right) r}\right)
$$

where

$$
c_{1}=\frac{3\left[(1-\gamma)\left(\beta_{1}\right)-6\left(C_{2}(m, \lambda, k, \gamma)\right)\left(\alpha_{1}\right)-C_{3}(m, \lambda, k, \gamma)\left(\beta_{1}\right)\right]+c_{1}^{*}}{6(1-\gamma)\left(\beta_{1}\right)},
$$

$$
\begin{aligned}
c_{1}^{*}= & \left\{3\left[(1-\gamma)\left(\beta_{1}\right)-6 C_{2}(m, \lambda, k, \gamma)\left(\alpha_{1}\right)-C_{3}(m, \lambda, k, \gamma)\left(\beta_{1}\right)\right]^{2}\right. \\
& \left.+72 C_{2}(m, \lambda, k, \gamma)(1-\gamma)\left(\alpha_{1}\right)\left(\beta_{1}\right)\right\}^{\frac{1}{2}}
\end{aligned}
$$$$
\text { and } r_{1}=\frac{-6(1-c)\left(C_{2}(m, \lambda, k, \gamma)\left(\alpha_{1}\right)\right)+r_{1}^{*}}{6 c(1-c)(1-\gamma)\left(\beta_{1}\right)} \text {, }
$$

$r_{1}^{*}=\left\{36(1-c)^{2}\left(C_{2}(m, \lambda, k, \gamma)\right)^{2}\left(\alpha_{1}^{2}\right)+12 c^{2}(1-c)(1-\gamma) C_{3}(m, \lambda, k, \gamma)\left(\beta_{1}^{2}\right)\right\}^{\frac{1}{2}}$.

The proof is omitted.

Theorem 4.7 Let the function $f(z)$ defined by (18) be in the class $k-\mathrm{Y} \Sigma \mathrm{T}_{s}\left(c, \lambda, \alpha_{1}, \beta_{1}, \gamma, t\right)$. Then, for $0 \leq r<1$,

$\left|f_{3}^{\prime}\left(r e^{i \theta}\right)\right| \geq 1-\frac{c(1-\gamma)\left(\beta_{1}\right)}{C_{2}(m, \lambda, k, \gamma)\left(\alpha_{1}\right)} r-\frac{(1-c)(1-\gamma)}{C_{3}(m, \lambda, k, \gamma)} r^{2}$

with equality for $f_{3}^{\prime}(z)$ at $z=r$ and

$$
\left|f_{3}^{\prime}\left(r e^{i \theta}\right)\right| \leq \max \left\{\max _{\theta}\left|f_{3}^{\prime}\left(r e^{i \theta}\right)\right|,-f_{4}^{\prime}(-r)\right\}
$$

where $\max _{\theta}\left|f_{3}^{\prime}\left(r e^{i \theta}\right)\right|$ is given by Lemma 4.6.

\section{Radii of Starlikeness and Convexity}

Theorem 5.1 Let the function $f(z)$ defined by (18) be in the class $k-\mathrm{Y \Sigma T}_{s}\left(c, \lambda, \alpha_{1}, \beta_{1}, \gamma, t\right)$. Then $f(z)$ is starlike of order $\rho \quad(0 \leq \rho<1)$ in the disc $|z|=r_{1}(c, \rho, m, \lambda, k, \gamma)$, where $r_{1}(c, \rho, m, \lambda, k, \gamma)$ is the largest value for which

$$
\begin{aligned}
& \frac{c(1-\gamma)(2-\rho)\left(\beta_{1}\right)}{C_{2}(m, \lambda, k, \gamma)(\alpha 1)} r+\frac{(1-\gamma)(1-c)(n-\rho)}{C_{n}(m, \lambda, k, \gamma)} r n-1 \\
& \leq 1-\rho \quad(n \geq 3) .
\end{aligned}
$$

The result is sharp with the extremal function

$$
f_{n}(z)=z-\frac{c(1-\gamma)\left(\beta_{1}\right)}{C_{2}(m, \lambda, k, \gamma)\left(\alpha_{1}\right)} z^{2}-\frac{(1-\gamma)(1-c)}{C_{n}(m, \lambda, k, \gamma)} z^{n},
$$

Proof. It suffices to show that

$$
\left|\frac{z f^{\prime}(z)}{f(z)}-1\right| \leq 1-\rho \quad(0 \leq \rho<1)
$$

for $|z|=r_{1}(c, \rho, m, \lambda, k, \gamma)$. We note that

$$
\begin{aligned}
\left|\frac{z f^{\prime}(z)}{f(z)}-1\right| & \leq \frac{\frac{c(1-\gamma)\left(\beta_{1}\right)}{C_{2}(m, \lambda, k, \gamma)\left(\alpha_{1}\right)} r+\sum_{n=3}^{\infty}(n-1) a_{n} r^{n-1}}{1-\frac{c(1-\gamma)\left(\beta_{1}\right)}{C_{2}(m, \lambda, k, \gamma)\left(\alpha_{1}\right)} r-\sum_{n=3}^{\infty} a_{n} r^{n-1}} \\
& \leq 1-\rho,
\end{aligned}
$$

$|z| \leq r$ if and only if

$$
\frac{c(1-\gamma)(2-\rho)\left(\beta_{1}\right)}{C_{2}(m, \lambda, k, \gamma)\left(\alpha_{1}\right)} r+\sum_{n=3}^{\infty}(n-\rho) a_{n} r^{n-1} \leq 1-\rho
$$

Since $f(z)$ is in the class $k-\mathrm{Y} \Sigma \mathrm{T}_{s}\left(c, \lambda, \alpha_{1}, \beta_{1}, \gamma\right)$, from (14) we may take

$$
a_{n}=\frac{(1-c)(1-\gamma)}{C_{n}(m, \lambda, k, \gamma)} \mu_{n} \quad(n=3,4,5, \ldots)
$$

where $\mu_{n} \geq 0$ and $\sum_{n=3}^{\infty} \mu_{n} \leq 1$.

For each fixed $r$, we choose the positive integer

$n_{0}=n_{0}(r)$ for which $\frac{\left(n_{0}-\rho\right)}{C_{n_{0}}(m, \lambda, k, \gamma)}$ is maximal. Then it

follows that

$$
\sum_{n=3}^{\infty}(n-\rho) a_{n} r^{n-1} \leq \frac{(1-c)(1-\gamma)\left(n_{0}-\rho\right)}{C_{n_{0}}(m, \lambda, k, \gamma)} r^{n_{0}-1} .
$$

Hence $f(z)$ is starlike is of order $\rho$ in $|z|=r_{1}(c, \rho, m, \lambda, k, \gamma)$ provided that $\frac{c(1-\gamma)(2-\rho)\left(\beta_{1}\right)}{C_{2}(m, \lambda, k, \gamma)\left(\alpha_{1}\right)} r+\frac{(1-\gamma)(1-c)\left(n_{0}-\rho\right)}{C_{n_{0}}(m, \lambda, k, \gamma)} r^{n_{0}-1} \leq 1-\rho$.

We find the value $r_{1}=r_{1}(c, \rho, m, \lambda, k, \gamma)$ and the corresponding integer $n_{0}\left(r_{0}\right)$ so that

$$
\frac{c(1-\gamma)(2-\rho)\left(\beta_{1}\right)}{C_{2}(m, \lambda, k, \gamma)\left(\alpha_{1}\right)} r_{0}+\frac{(1-\gamma)(1-c)\left(n_{0}-\rho\right)}{C_{n_{0}}(m, \lambda, k, \gamma)} r_{0}^{n_{0}-1}=1-\rho .
$$

Then this value $r_{0}$ is the radius of starlikeness of order $\rho$ for functions $f(z)$ belonging to the class $k-\mathrm{Y} \Sigma \mathrm{T}_{s}\left(c, \lambda, \alpha_{1}, \beta_{1}, \gamma, t\right)$.

In similar manner, we can prove the following theorem concerning the radius of convexity of $\rho$ for functions in the class $k-\mathrm{Y} \Sigma \mathrm{T}_{s}\left(c, \lambda, \alpha_{1}, \beta_{1}, \gamma, t\right)$.

Theorem 5.2 Let the function $f(z)$ defined by (18) be in the class $k-\mathrm{Y} \Sigma \mathrm{T}_{s}\left(c, \lambda, \alpha_{1}, \beta_{1}, \gamma, t\right)$. Then $f(z)$ is convex of order $\rho, \quad(0 \leq \rho<1)$ in the disc 


\section{International Journal of Science and Research (IJSR) \\ ISSN (Online): 2319-7064}

Index Copernicus Value (2016): 6.14 | Impact Factor (2015): 6.391

$|z|=r_{2}(c, \rho, m, \lambda, k, \gamma)$, where $r_{2}(c, \rho, m, \lambda, k, \gamma)$ is the largest value for which

$$
\begin{aligned}
& \frac{2 c(1-\gamma)(2-\rho)\left(\beta_{1}\right)}{C_{2}(m, \lambda, k, \gamma)\left(\alpha_{1}\right)} r+\frac{(1-\gamma)(1-c) n(n-\rho)}{C_{n}(m, \lambda, k, \gamma)} r^{n-1} \\
& \leq 1-\rho \quad(n \geq 3) .
\end{aligned}
$$

The result is sharp for the function $f(z)$ given by (55).

\section{References}

[1] A. W. Goodman, On uniformly starlike functions, J. Math. Anal. Appl. 155, no. 2, 64-370, 1991.

[2] A. W. Goodman, On uniformly convex functions, Ann. Polon. Math. 56, no. 1, 87-92, 1991.

[3] Ma, Wan Cang; Minda, David., Uniformly convex functions, Ann. Polon. Math. 57, no. 2, 165-175, 1992.

[4] F. Ronning, On starlike functions associated with parabolic regions, Ann. Univ. Mariae Curie- Skodowska Sect., A 45, 117-122, 1992.

[5] K. Sakaguchi, On a certain univalent mapping, J. Math. Soc., Japan, 11, 72-75, 1959.

[6] Owa, Shigeyoshi; Sekine, Tadayuki; Yamakawa, Rikuo., On Sakaguchi type functions, Appl. Math. Comput. 187, no. 1, 356-361, 2007.

[7] C. Selvaraj and K. R. Karthikeyan, Differential sandwich theorems for certain subclasses of analytic functions, Math. Commun.,13, no. 2, 311-319, 2008.

[8] J. Dziok and H. M. Srivastava, Classes of analytic functions associated with the generalized hypergeometric function, Appl. Math. Comput., 103, no. 1, 1-13, 1999.

[9] G. S. Salagean, Subclasses of univalent functions, Complex analysis fifth Romanian- Finnish seminar, Part 1 (Bucharest, 1981), 362-372, Lecture Notes in Math., 1013, Springer, Berlin, 1983.

[10]M. K. Aouf, H. E. Darwish, Fixed coefficients for certain class of analytic functions with negative coefficients, Commun. Fac. Sci. Univ. Ank. Ser. A1 Math. Stat. 45, no. 1-2, 37-44, 1997.

[11] M. K. Aouf, A. A. Al-Dohiman, Fixed coefficients for certain subclass of univalent functions with negative coefficients, Punjab Univ. J. Math. (Lahore) 37, 129-147, 2005.

[12] S. M. Khairnar, N. H. More, A subclass of uniformly convex functions and a corresponding subclass of starlike functions with fixed second coefficient defined by Carlson and Shaffer operator, Int. Math. Forum 5, no. 37-40, 1839-1848, 2010.

[13]H. E. Darwish, On a subclass of uniformly convex functions with fixed second coefficient, Demonstratio Math. 41, no. 4, 791-803, 2008.

[14] G. Murugusundaramoorthy, N. Magesh, A new subclass of uniformly convex functions and a corresponding subclass of starlike functions with fixed second coefficient, JIPAM. J. Inequal. Pure Appl. Math. 5, no. 4, Article 85, 10 pp, 2004.

[15] Murat Caglar and Halit Orhan, On Coefficient Estimates and Neighborhood Problem for Sakaguchi type Functions, 2012, arxiv: 1204.4546v1.
[16]H. Silverman, E.M. Silvia, Fixed coefficients for subclasses of starlike functions, Houston J. Math. 7, no. 1, 129-136, 1981.

[17]C. Selvaraj, K. A. Selvakumaran, New classes of $k$ uniformly convex and starlike functions with respect to other points, Acta Math. Univ. Comenian. (N.S.) 78 , no. 1, 103-114, 2009.

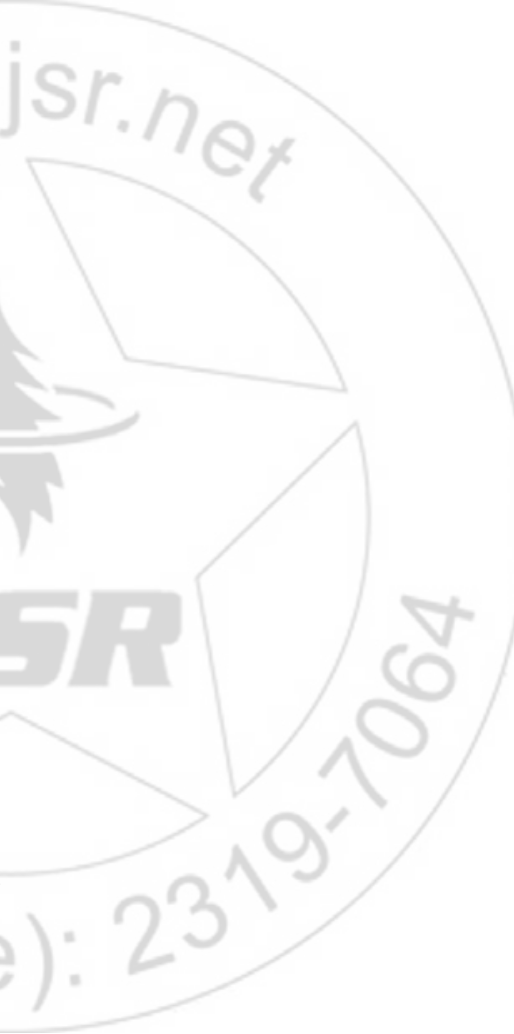

